\title{
Robust Admissibilization of Descriptor Systems by Static Output-Feedback: An LMI Approach
}

\author{
M. Chaabane, ${ }^{1}$ F. Tadeo, ${ }^{2}$ D. Mehdi, ${ }^{3}$ and M. Souissi ${ }^{1}$ \\ ${ }^{1}$ Research Unit of Industrial Processes Control (UCPI), National Engineering School of Sfax, (ENIS), \\ Route Soukra Km 3.5, BP 1173, 3038 Sfax, Tunisia \\ ${ }^{2}$ Departamento de Ingenieria de Sistemas y Automatica, Universidad de Valladolid, 47011 Valladolid, Spain \\ ${ }^{3}$ Laboratory of Automatic and Industrial Informatics (LAII), Higher School of Engineers of Poitiers (ESIP), \\ 40 Avenue du Recteur Pineau, 86022 Poitiers Cedex, France
}

Correspondence should be addressed to F. Tadeo, fernando@autom.uva.es

Received 23 November 2010; Revised 7 February 2011; Accepted 10 March 2011

Academic Editor: J. Rodellar

Copyright (c) 2011 M. Chaabane et al. This is an open access article distributed under the Creative Commons Attribution License, which permits unrestricted use, distribution, and reproduction in any medium, provided the original work is properly cited.

The problem of the stabilization of descriptor systems in continuous-time via static outputfeedback is studied in this paper and an approach to solve it is proposed. For this, sufficient conditions are derived for the closed-loop system to be admissible (i.e., stable, regular, and impulsefree). These conditions are expressed in terms of a strict Linear Matrix Inequality (LMI); so they are tractable using numerical computations. The proposed controller design methodology is based on two steps: the first is dedicated to synthesizing a classical state-feedback controller, which is used as the initial value for the second step, which uses an LMI problem to obtain static output-feedback controllers that give admissibility. Finally, a numerical example is given to illustrate the results.

\section{Introduction}

Descriptor systems result from a convenient modeling process (see [1-3]). It is fair to say that descriptor system models give a more complete class of dynamical models than the state variable systems, as a descriptor form also includes information about static constraints. Applications of descriptor systems can be found in various fields such as electrical circuit networks [1], robotics [4], and economics [5].

In recent years, considerable effort has been devoted to the analysis of stability (see [3, 6-8]), the development of stabilization techniques, and the study of robustness of descriptor systems (see [9-16]).

Many results concerning LMI-based control use LMIs, but with additional equality constraints, which, unfortunately, often cause numerical problems in computation (see $[10,17-19])$. This motivated the authors, following [9], to express the LMI conditions for the analysis of admissibility and controller synthesis [6] in terms of a strict LMI 
(i.e., LMI without any additional equality constraints), so as to be more tractable and so the numerical solution will be more reliable when using the available LMI software solvers.

In this paper, our main contribution is an approach for the synthesis of static outputfeedback controllers, based on solving a strict LMI (i.e., without additional constraints) for a problem of admissibilization for Descriptor Systems. Uncertainty in Descriptor Systems is a serious issue [9]; so an extension of the main results for uncertain singular systems is also provided. In order to give the conditions of admissibility in LMI form, some relaxation variables are introduced.

Robust control of linear state space systems has received much attention over the last few decades and various aspects and approaches for analysis and control design, for linear systems with uncertain parameters, have been investigated (see [20, 21]). It must be pointed out that many important results on quadratic stability and stabilization approaches have been proposed in the literature. For example, the quadratic stability and stabilization problems have been studied, but they are usually characterized by the determination of a unique Lyapunov matrix (see [22]), which gives the approach an inherent conservatism. Recently, the Parameter Dependent Lyapunov (PDL) approach has been introduced to reduce the conservatism of the quadratic approach. This PDL approach consists of expressing the Lyapunov matrix as a function of uncertainty, with the use of some additional variables, which allows a significant reduction of the conservatism [23]. Thus, the PDL approach is used in this paper to solve the robust static output-feedback admissibility problem, for the descriptor systems case.

This paper is organized as follows. Section 2 gives the problem formulation and some preliminary definitions. Section 3 gives the main results for solving the static feedback problems of nominal descriptor systems, whereas Section 4 presents an extension of the main results for uncertain singular systems. Section 5 presents an illustrative example. Finally, Section 6 gives some conclusions.

\section{Preliminaries}

Consider the following descriptor system in continuous-time:

$$
E \dot{x}(t)=A x(t),
$$

where $x(t) \in \mathbb{R}^{n}$ is the state, the matrix $E$ may be singular (so we assume that $\operatorname{rank}(E)=r \leq$ $n)$, and $A$ is a known real constant matrix of appropriate dimension.

Definition 2.1 (see [1]). The pair $(E, A)$ is said to be $\operatorname{regular}$ if $\operatorname{det}(s E-A)$ is not identically zero.

Definition 2.2 (see [1]). The pair $(E, A)$ is said to be impulse-free if $\operatorname{deg}(\operatorname{det}(s E-A))=\operatorname{rank}(E)$.

In the rest of the paper, the notation is standard, unless specified otherwise. $L>0$ $(L<0)$ means that the matrix $L$ is a symmetric and positive defined matrix (symmetric and negative defined). For a square matrix $X, \operatorname{Sym}\{X\}$ is defined as $\operatorname{Sym}\{X\}=X+X^{\top}$, with $T$ denoting the transpose. 
It is worth noting that the stability properties for conventional systems are no longer sufficient for singular systems, unless they are completed by the regularity condition and the absence of impulses, which leads to the introduction of the notion of admissibility.

Definition 2.3 (see $[1,10]$ ). The descriptor system (2.1) is said to be admissible if it is regular, impulse-free, and Hurwitz stable.

In order to characterize the admissibility of a singular system, let us recall that for a pair $(E, A)$ there exists a transformation pair $(U, V)$ such that

$$
\bar{E}=U E V=\left[\begin{array}{ll}
I & 0 \\
0 & 0
\end{array}\right], \quad \bar{A}=U A V=\left[\begin{array}{ll}
\bar{A}_{11} & \bar{A}_{12} \\
\bar{A}_{21} & \bar{A}_{22}
\end{array}\right]
$$

To solve the static output-feedback admissibilitation problem, the following theorem is introduced.

Theorem 2.4 (see [6]). The continuous singular system $(E, A)$ is admissible if and only if there exist three matrices $X, Y$, and $Z$ such that

$$
\begin{gathered}
E X E^{\top}+\operatorname{Sym}\left\{E^{\dagger} Z\right\}>0, \\
\operatorname{Sym}\left\{A\left(X E^{\top}+E^{\perp} Y\right)\right\}<0,
\end{gathered}
$$

with $E^{\dagger} \doteq U^{-1}(I-U E V) U$ and $E^{\perp} \doteq V(I-U E V) U$, that fulfil $E E^{\perp}=0$ and $E^{\dagger} E=0$.

It must be pointed out that if the singular system defined by the pair $(E, A)$ is admissible, then its dual, defined by the pair $\left(E^{\top}, A^{\top}\right)$, is also admissible; so Theorem 2.4 can also be written as follows.

Corollary 2.5. The continuous singular system is admissible if and only if there exist matrices $X, Y$ and $Z$ such that

$$
\operatorname{Sym}\left\{A^{\top}\left(X E+E^{\ddagger} Y\right)\right\}<0,
$$

with $E^{\ddagger} \doteq U^{\top}(I-U E V) U^{-\top}$ (which fulfills $E^{\top} E^{\ddagger}=0$ ).

\section{Admissibilization by Static Output-Feedback Control}

In this section we address the problem of admissibility by static output-feedback for the descriptor system given by

$$
\begin{gathered}
E \dot{x}(t)=A x(t)+B u(t), \\
\mathrm{y}(t)=C x(t)
\end{gathered}
$$

where $x(t) \in \mathbb{R}^{n}$ is the state, $u(t) \in \mathbb{R}^{m}$ is the control input, and $y(t) \in \mathbb{R}^{p}$ is the output. 
$E, A, B$, and $C$ are real matrices that, in this section, are assumed to be known and constant (this restriction will be lifted in Section 3).

The control law given by a static output-feedback is then

$$
u(t)=K y(t)
$$

where the constant gain $K$, of appropriate dimensions, is computed in such a way that the (singular) closed-loop system is admissible.

As the goal of this paper is to find gains $K$ such that the closed-loop system is admissible, we present the main result in this section: a solution of admissibilization problem by static output-feedback, where the solvability will be given by some LMI conditions. This approach is summarized by Lemmas 3.1 and 3.2. If the system contains polytopic uncertainties, the results can be extended to the robust static output-feedback admissibility problem, which is dealt with in Section 4.

For this, we note that the descriptor system (3.1) in closed-loop under feedback (3.3) is given by

$$
E \dot{x}(t)=(A+B K C) x
$$

This closed-loop system is admissible if the following inequality is verified:

$$
\operatorname{Sym}\left\{(A+B K C)^{\top}\left(X E+E^{\ddagger} Y\right)\right\}<0,
$$

where the matrix $X$ satisfies condition (2.3).

From this result, the static output-feedback controller can be synthesized, guaranteeing that the closed-loop system (3.4) is admissible. We will propose a solution in two steps. The first step is devoted to establishing a relation between a classical state feedback controller design and static output feedback. In the second step, we include some relaxed variables and so the design of the controller gain is formulated as an LMI problem. The following lemmas give LMI-based conditions for the solvability of the problem.

Lemma 3.1. (relation between state-feedback and static output-feedback). The following statements are equivalent.

(1) There exist matrices $X, Y$, and $Z$, a state-feedback gain $K_{0}$, and an output-feedback gain $K$ such that

$$
\begin{aligned}
& \operatorname{Sym}\left\{(A+B K C)^{\top}\left(X E+E^{\ddagger} Y\right)\right\}<0, \\
& \operatorname{Sym}\left\{\left(A+B K_{o}\right)^{\top}\left(X E+E^{\ddagger} Y\right)\right\}<0 .
\end{aligned}
$$


(2) There exist matrices $X, Y$, and $Z$, a nonsingular matrix $G$, a state-feedback gain $K_{o}$, and an output-feedback gain $\mathrm{K}$ such that

$$
\left[\begin{array}{cc}
\operatorname{Sym}\left\{\left(A+B K_{o}\right)^{\top}\left(X E+E^{\ddagger} Y\right)\right\} & \left(K C-K_{o}\right)^{\top} G^{\top}+\left(X E+E^{\ddagger} Y\right)^{\top} B \\
G\left(K C-K_{o}\right)+B^{\top}\left(X E+E^{\ddagger} Y\right) & -G+G^{\top}
\end{array}\right]<0,
$$

where the matrix $X$ satisfies the condition (2.3):

$$
E X E^{\top}+\operatorname{Sym}\left\{E^{\dagger} Z\right\}>0
$$

Proof. First, note that inequality (3.7) can be written as follows:

$$
\phi+\operatorname{Sym}\left\{\left[\begin{array}{l}
0 \\
I
\end{array}\right] G[S-I]\right\}<0
$$

with $S=K C-K_{o}$ and

$$
\phi=\left[\begin{array}{cc}
\operatorname{Sym}\left\{\left(A+B K_{o}\right)^{\top}\left(X E+E^{\ddagger} Y\right)\right\} & \left(X E+E^{\ddagger} Y\right)^{\top} B \\
B^{\top}\left(X E+E^{\ddagger} Y\right) & 0
\end{array}\right] .
$$

A direct application of the Elimination Lemma [24] allows us to state that the above condition is equivalent to

$$
\begin{gathered}
{\left[\begin{array}{ll}
I & 0
\end{array}\right] \phi\left[\begin{array}{l}
I \\
0
\end{array}\right]=\operatorname{Sym}\left\{\left(A+B K_{o}\right)^{\top}\left(X E+E^{\ddagger} Y\right)\right\}<0,} \\
{\left[\begin{array}{ll}
I & S^{\top}
\end{array}\right] \phi\left[\begin{array}{l}
I \\
S
\end{array}\right]=\operatorname{Sym}\left\{(A+B K C)^{\top}\left(X E+E^{\ddagger} Y\right)\right\}<0,}
\end{gathered}
$$

to obtain the inequalities (3.6) of Lemma 3.1.

Lemma 3.1 shows the existence of a relation between the state-feedback and the output-feedback, in the sense that they have the same Lyapunov matrix verifying (2.3). Thus, the output-feedback gain can be calculated by determining a solution of the inequality (3.7). For this, we introduce supplementary variables that allow us to deduct a sufficient condition for the development of the gain of the output-feedback, given by the following lemma.

Lemma 3.2. For a descriptor system defined by (3.4), the following are equivalent.

(1) There exist matrices $X$ and $Y$, a nonsingular matrix $G$, a state-feedback gain $K_{o}$, and an output-feedback gain K such that

$$
\left[\begin{array}{cc}
\operatorname{Sym}\left\{\left(A+B K_{o}\right)^{\top}\left(X E+E^{\ddagger} Y\right)\right\} & \left(K C-K_{o}\right)^{\top} G^{\top}+\left(X E+E^{\ddagger} Y\right)^{\top} B \\
G\left(K C-K_{o}\right)+B^{\top}\left(X E+E^{\ddagger} Y\right) & -G-G^{\top}
\end{array}\right]<0 .
$$


(2) There exist matrices $X, Y, Z, F_{1}, F_{2}, F_{3}$, and $L$, and a nonsingular matrix $G$, such that the following condition is satisfied for all given $K_{o}$ :

$$
H+\operatorname{Sym}\left\{\left[\begin{array}{l}
F_{1} \\
F_{2} \\
F_{3}
\end{array}\right]\left[\begin{array}{lll}
A_{o} & B & -I
\end{array}\right]\right\}<0,
$$

with $A_{0}=A+B K_{o}$, and

$$
H=\left[\begin{array}{ccc}
0 & \left(L C-G K_{o}\right)^{\top} & \left(X E+E^{\ddagger} Y\right)^{\top} \\
L C-G K_{o} & -G-G^{\top} & 0 \\
X E+E^{\ddagger} Y & 0 & 0
\end{array}\right]
$$

and where matrix $X$ satisfies condition (2.3): $E X E^{\top}+\operatorname{Sym}\left\{E^{\dagger} Z\right\}>0$.

Thus, if the LMI (3.13) is feasible, then the singular closed-loop system with the static outputfeedback gain $K=G^{-1} L$ is admissible.

Proof. According to the Elimination Lemma [24], condition (3.12) holds if and only if there exists a matrix $F$ satisfying the following condition:

$$
\phi+\operatorname{Sym}\{F Q\}<0,
$$

with

$$
\phi=\left[\begin{array}{ccc}
0 & \left(L C-G K_{o}\right)^{\top} & X E^{\top}+E^{\perp} Y \\
L C-G K_{o} & -\left(G+G^{\top}\right) & 0 \\
\left(X E^{\top}+E^{\perp} Y\right)^{\top} & 0 & 0
\end{array}\right],
$$

$Q=\left[\begin{array}{lll}A_{0} & B & -I\end{array}\right]^{\top}$ and $F$ partitioned as $F=\left[\begin{array}{ll}F_{1} F_{2} F_{3}\end{array}\right]^{\top}$, with $F_{3}$ nonsingular.

Making the change of variable $L=G K$ in this condition yields directly condition (3.13), which completes the proof.

\section{Robust Admissibilization by Static Output-Feedback}

In this section, we consider the output-feedback admissibilization problem for systems containing uncertainties, which are assumed to lie in a bounded convex domain. The results developed in Section 3 will be applied to solving the robust static output-feedback admissibility problem in this section. 
Assume that the uncertain descriptor system is characterized by

$$
\begin{gathered}
E \dot{x}(t)=A(\alpha) x(t)+B(\alpha) u(t), \\
y(t)=C(\alpha) x(t),
\end{gathered}
$$

where the matrices $A(\alpha), B(\alpha)$, and $C(\alpha)$ are given as follows:

$$
A(\alpha) \doteq \sum_{i=1}^{N} \alpha_{i} A_{i}, \quad B(\alpha) \doteq \sum_{i=1}^{N} \alpha_{i} B_{i}, \quad C(\alpha) \doteq \sum_{i=1}^{N} \alpha_{i} C_{i}
$$

with

$$
\alpha \doteq\left[\alpha_{1} \cdots \alpha_{N}\right]^{\top}, \quad \sum_{i=1}^{N} \alpha_{i}=1, \quad \alpha_{i} \geq 0, \text { for } i=1, \ldots, N
$$

That is, the system matrices $[A(\alpha) B(\alpha) C(\alpha)]$ belong to a polytope whose vertices are $\left[\begin{array}{lll}A_{i} & B_{i} & C_{i}\end{array}\right]$ for $i=1, \ldots, N$.

The output-feedback stabilization of system (4.1) consists of an adequate choice of a feedback gain $K$ such that the closed-loop system

$$
E \dot{x}(t)=(A(\alpha)+B(\alpha) K C(\alpha)) x(t)
$$

is admissible for every $\alpha$ satisfying (4.4).

Then the admissibilization problem has a solution if there exists a static outputfeedback $K$ such that, for every $i$, the closed-loop system characterized by $\left[E A_{i} B_{i} C_{i}\right]$ is admissible. The solution of this problem is summarized by the next Theorem 4.1.

Theorem 4.1. For the uncertain descriptor system characterized by $E, A(\alpha)$, and $B(\alpha)$, as given in (4.1)-(4.4), if there exist matrices $X_{i}, Y_{i}, Z, F_{1}, F_{2}, F_{3}$, and $L$, and a nonsingular matrix $G$ such that the following LMIs are feasible for given $K_{o}$

$$
H_{i}+\operatorname{Sym}\left\{\left[\begin{array}{l}
F_{1} \\
F_{2} \\
F_{3}
\end{array}\right]\left[\begin{array}{lll}
A_{i}+B_{i} K_{o} & B_{i} & -I
\end{array}\right]\right\}<0, \quad i=1, \ldots, N
$$

with

$$
H_{i}=\left[\begin{array}{ccc}
0 & \left(L C_{i}-G K_{o}\right)^{\top} & \left(X_{i} E+E^{\ddagger} Y_{i}\right)^{\top} \\
L C_{i}-G K_{o} & -G-G^{\top} & 0 \\
X_{i} E+E^{\ddagger} Y_{i} & 0 & 0
\end{array}\right],
$$


and matrices $X_{i}$ satisfying condition (2.3), then, an admissible static output-feedback gain is given by

$$
K=G^{-1} L
$$

Proof. First, multiply each condition in (4.6) by $\alpha_{i}$, as defined in (4.4) and then sum them up (from $i=1$ to $i=N$ ). Then, substituting in the result $\sum_{i=1}^{N} \alpha_{i} X_{i}$ by $X$ and $\sum_{i=1}^{N} \alpha_{i} Y_{i}$ by $Y$ gives directly the condition (3.13); so applying Lemma 3.2 completes the proof.

\section{Illustrative Example}

Example 5.1. Consider a descriptor system in continuous-time as in (2.1), described by the following system matrices [10]:

$$
E=\left[\begin{array}{lll}
1 & 0 & 0 \\
0 & 1 & 0 \\
0 & 0 & 0
\end{array}\right], \quad A=\left[\begin{array}{ccc}
0 & 1 & 1 \\
-1 & 3 & 0 \\
0 & 0 & 0
\end{array}\right], \quad B=\left[\begin{array}{cc}
0 & 0.2 \\
1 & 0 \\
-0.1 & 1
\end{array}\right], \quad C=\left[\begin{array}{ccc}
1 & 0 & 1 \\
0.5 & 1 & 0
\end{array}\right] \text {. }
$$

It can be seen that the open-loop system is not admissible: poles are located at 0.3820 and 2.6180. To make this system admissible by output-feedback, it is first necessary to compute a state-feedback gain that makes the closed-loop system admissible. For example, using the method described in [6], we computed

$$
K_{o}=\left[\begin{array}{ccc}
0.3864 & -4.5658 & -0.4861 \\
-0.9965 & -1.4100 & -1.8631
\end{array}\right] \text {. }
$$

Then, the LMIs in Lemma 3.2 are feasible. One solution (given by the LMI toolbox in Matlab) is

$$
\begin{aligned}
& X=\left[\begin{array}{ccc}
0.8457 & 0.0958 & -0.5277 \\
0.0958 & 0.7172 & 24.9240 \\
-0.5277 & 24.9240 & 0
\end{array}\right], \quad Y=\left[\begin{array}{ccc}
0 & 0 & 0 \\
0 & 0 & 0 \\
0.9287 & -24.7339 & 0.7066
\end{array}\right], \\
& G=\left[\begin{array}{cc}
0.2884 & -0.2837 \\
-0.2883 & 0.8003
\end{array}\right], \quad L=\left[\begin{array}{cc}
0.6965 & -1.0927 \\
-1.1116 & 0.1593
\end{array}\right], \quad Z=\left[\begin{array}{ccc}
0 & 0 & 0 \\
0 & 0 & 0 \\
0 & 0 & 0.4998
\end{array}\right] \text {, }
\end{aligned}
$$

which gives the static output-feedback gain

$$
K=G^{-1} L=\left[\begin{array}{cc}
1.6243 & -5.5642 \\
-0.8039 & -1.8051
\end{array}\right] \text {. }
$$


It can be seen that this feedback gain renders the resulting closed-loop system admissible, with finite closed-loop poles located at -1.1159 and -5.2701 .

\section{Conclusion}

The problem of robust admissibilization for continuous time descriptor systems has been studied. Some conditions have been derived to ensure the admissibility of the closed loop via a static output-feedback control law. These conditions are expressed in terms of strict Linear Matrix Inequalities, without additional equality constraints. Based on this result, a robust admissible output-feedback control law is proposed for continuous descriptor systems with polytopic uncertainty. The proposed method consists of two steps. The first step is devoted to a classical state feedback controller design, whereas the second one is the solution of the LMI problem. A numerical example is provided to illustrate the usefulness of the proposed methodology.

\section{Acknowledgment}

This work was funded by the AECI projects A/023792/09 and A/030410/10, and by MiCInn DPI2010-21589-C05-05.

\section{References}

[1] L. Dai, Singular Control Systems, vol. 118 of Lecture Notes in Control and Information Sciences, Springer, Berlin, Germany, 1989.

[2] G.-R. Duan, Analysis and Design of Descriptor Linear Systems, vol. 23 of Advances in Mechanics and Mathematics, Springer, New York, NY, USA, 2010.

[3] F. L. Lewis, "A survey of linear singular systems," Circuits, Systems, and Signal Processing, vol. 5, no. 1, pp. 3-36, 1986.

[4] J. K. Mills and A. A. Goldenberg, "Force and position control of manipulators during constrained motion tasks," IEEE Transactions on Robotics and Automation, vol. 5, no. 1, pp. 30-46, 1989.

[5] D. G. Luenberger, "Singular dynamic Loentief systems," Econometrica, vol. 45, pp. 991-994, 1977.

[6] M. Chaabane, O. Bachelier, M. Souissi, and D. Mehdi, "Stability and stabilization of continuous descriptor systems: an LMI approach," Mathematical Problems in Engineering, vol. 2006, Article ID 39367, 15 pages, 2006.

[7] J. Y. Ishihara and M. H. Terra, "On the Lyapunov theorem for singular systems," IEEE Transactions on Automatic Control, vol. 47, no. 11, pp. 1926-1930, 2002.

[8] J. Y. Ishihara and M. H. Terra, "On the Lyapunov theorem for singular systems," IEEE Transactions on Automatic Control, vol. 47, no. 11, pp. 1926-1930, 2002.

[9] F. A. Faria, E. Assunção, M. C. M. Teixeira, and R. Cardim, "Robust state-derivative feedback LMIbased designs for linear descriptor systems," Mathematical Problems in Engineering, vol. 2010, Article ID 927362, 15 pages, 2010.

[10] C. H. Kuo and C. H. Fang, "An LMI approach to admissibilization of uncertain descriptor systems via static output-feedback," in Proceedings of the American Control Conference, 2003.

[11] I. Masubuchi, "Output feedback controller synthesis for descriptor systems satisfying closed-loop dissipativity," Automatica, vol. 43, no. 2, pp. 339-345, 2007.

[12] V. Lovass-Nagy, D. L. Powers, and R. J. Schilling, “On regularizing descriptor systems by output feedback," IEEE Transactions on Automatic Control, vol. 39, no. 7, pp. 1507-1509, 1994.

[13] O. Rejichi, O. Bachelier, M. Chaabane, and D. Mehdi, "Robust root-clustering analysis in a union of subregions for descriptor systems," IET Control Theory \& Applications, vol. 2, no. 7, pp. 615-624, 2008.

[14] S. Xu and J. Lam, "Robust stability and stabilization of discrete singular systems: an equivalent characterization," IEEE Transactions on Automatic Control, vol. 49, no. 4, pp. 568-574, 2004.

[15] S. Xu, J. Lam, Y. Zou, and J. Li, "Robust admissibility of time-varying singular systems with commensurate time delays," Automatica, vol. 45, no. 11, pp. 2714-2717, 2009. 
[16] J. Yoneyama, "Robust stability for descriptor systems with time-varying delay," Applied Mathematical Sciences, vol. 4, no. 17-20, pp. 977-989, 2010.

[17] D. L. Chu and D. W. C. Ho, "Necessary and sufficient conditions for the output feedback regularization of descriptor systems," IEEE Transactions on Automatic Control, vol. 44, no. 2, pp. 405412, 1999.

[18] M. Inoue, T. Wada, M. Ikeda, and E. Uezato, “Stabilization of linear rime-varying descriptor systems by output feedback," in Proceedings of the European Control Conference (ECC '09), Budapest, Hungary, 2009.

[19] M. Yagoubi, "Static output feedback control design for descriptor systems," Journal of Control Science and Engineering, vol. 2010, Article ID 482561, 8 pages, 2010.

[20] C. B. Soh, C. S. Berger, and K. P. Dabke, "A simple approach to stabilization of discrete-time systems by output feedback," International Journal of Control, vol. 42, no. 6, pp. 1481-1490, 1985.

[21] A. Trofino-Neto, "Stabilization via static output feedback," IEEE Transactions on Automatic Control, vol. 38, no. 5, pp. 764-765, 1993.

[22] K. Zhou and P. Khargonekar, Robust and Optimal Control, Prentice Hall, Upper Saddle River, NJ, USA, 1996.

[23] J. Daafouz and J. Bernussou, "Parameter dependent Lyapunov functions for discrete time systems with time varying parametric uncertainties," Systems $\mathcal{E}$ Control Letters, vol. 43, no. 5, pp. 355-359, 2001.

[24] R. E. Skelton, T. Iwasaki, and K. M. Grigoriadis, A Unified Algebraic Approach to Linear Control Design, The Taylor \& Francis Systems and Control Book Series, Taylor \& Francis, London, UK, 1998. 


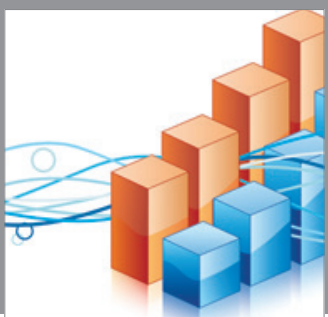

Advances in

Operations Research

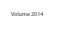

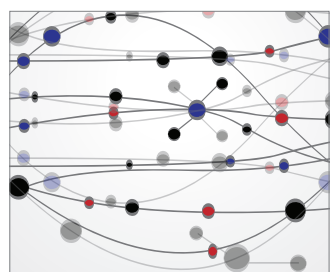

\section{The Scientific} World Journal
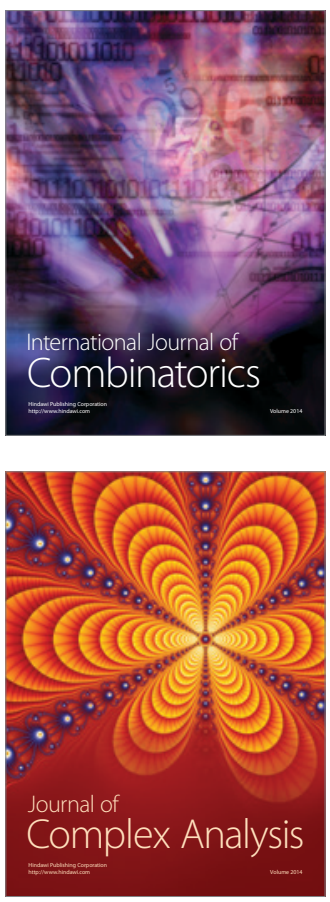

International Journal of

Mathematics and

Mathematical

Sciences
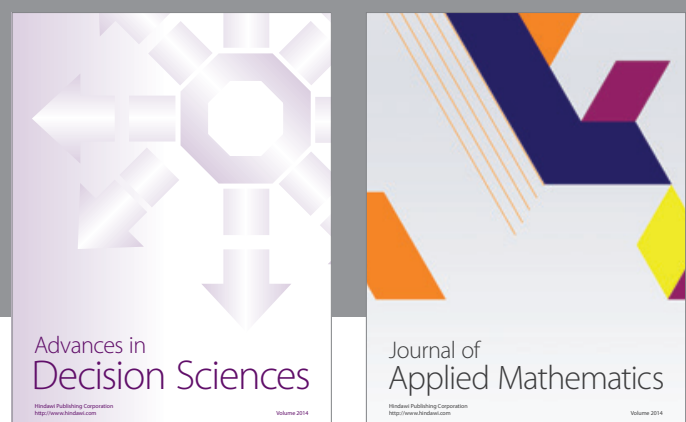

Journal of

Applied Mathematics
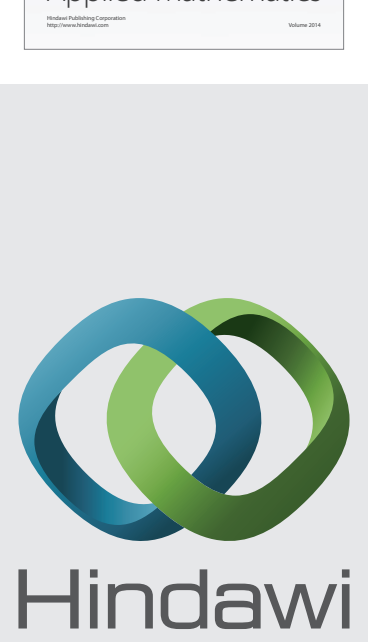

Submit your manuscripts at http://www.hindawi.com
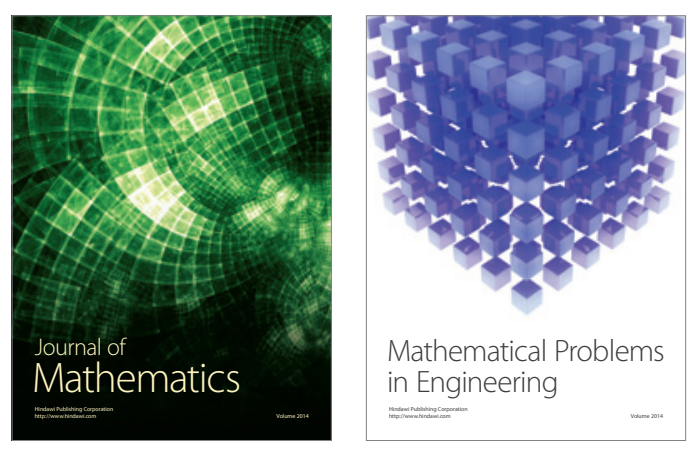

Mathematical Problems in Engineering
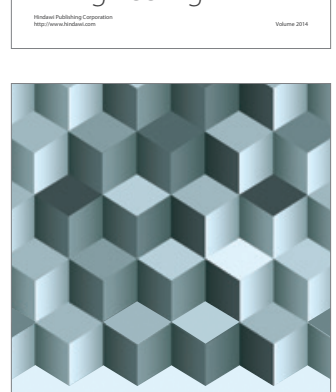

Journal of

Function Spaces
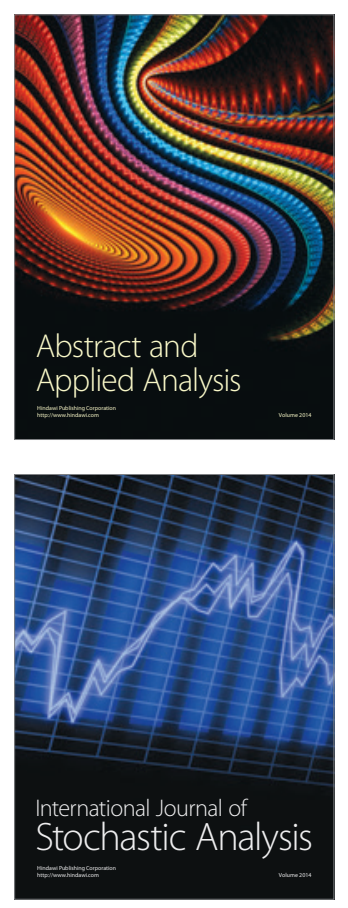

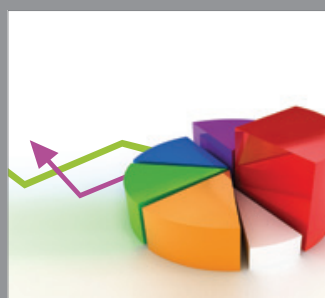

ournal of

Probability and Statistics

Promensencen
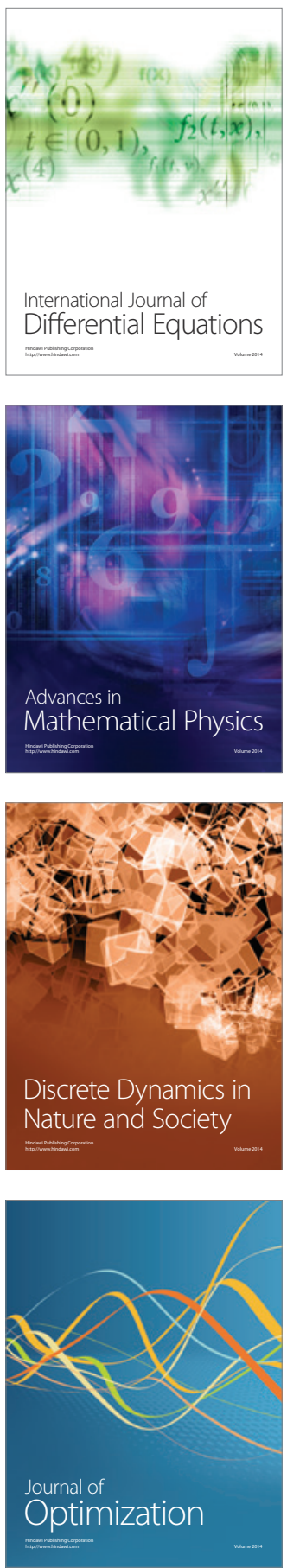